The Last Word

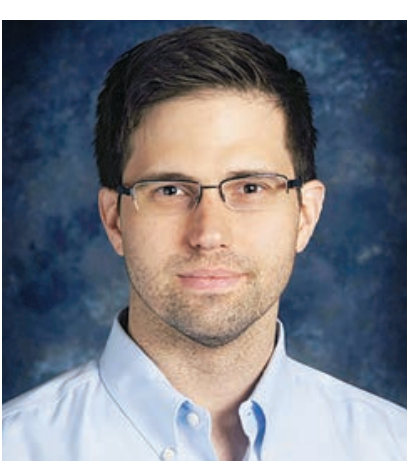

David R. Braxton, MD

David R. Braxton, MD, is an anatomic and clinical pathologist subspecializing in gastrointestinal, liver, and pancreatic diseases and molecular genetic pathology. He has experience in personalized medicine in the clinical and experimental research settings. Dr. Braxton's cancer research interests include identification of new biomarkers and the clinical significance of genomic profiles. He currently serves as the molecular pathology consultant for the Hoag Family Cancer Institute's Personalized Medicine Program. Hoag Family Cancer Institute is one of the largest community cancer programs in Southern California and has formed an alliance with USC Norris Comprehensive Cancer Center to expand access to subspecialized cancer care and clinical trials in Orange County.

\section{Acting on Rare Driver Alterations: Is Getting a Meaningful Response a Game of Chance or Scientific Rigor?}

\author{
David R. Braxton, MD
}

In this issue of JNCCN, Hamad et al (page 983) report an exceptional response to trastuzumab plus FOLFOX in a case of metastatic adenocarcinoma of the duodenum with ERRB2 (HER2/neu) amplification. This exceptional response is important to document for the oncology community as a whole, although it is difficult to attribute the complete pathologic response to trastuzumab alone, because it was used in combination with FOLFOX. Delineating the contribution of targeted approaches to pathologic response when used in combination with traditional chemotherapeutics is difficult outside the setting of a controlled trial Nevertheless, it illustrates that rare but actionable driver alterations can be identified in uncommon cancers that lack clinical treatment guidelines for targeted therapy.

The landscape of driver alterations in duodenal and ampullary carcinomas is incomplete at best. What we do know is that ERRB2 alterations have been reported with varying frequency in small bowel and ampullary adenocarcinomas, with missense mutations being the most frequent alteration in one study. ${ }^{1}$ Conversely, amplifications in ERBB2 are relatively rare in small bowel cancers and probably represent $<3 \%$ of putative driver alterations in this histology. 2,3 That ERRB2 alterations are the founder mutations in duodenal cancers has not been well established, and to what extent ERRB2 alterations are subject to intratumoral heterogeneity in these cancers is uncertain. If ERRB2 amplification is only present in a subclonal population, then targeting it would be unlikely to induce a complete pathologic response. So how did the authors overcome the many pitfalls in routine molecular biomarker testing and decide to take action and target this rare putative driver alteration?

First, no other driver mutation was found in the molecular profiling that was performed by a relatively modest gene panel that included BRAF, HRAS, KRAS, NRAS, and PIK3CA gene sequencing and ALK by immunohistochemistry. Second, ERRB2 amplification was found in both the primary and metastatic lesions. Therefore, the amplification did not have other drivers to compete with and was less likely to be a subclonal alteration. These findings lend some support for ERRB2 amplification as the major driver alteration in this particular case.

So, what factors are important in deciding that a putative driver alteration is actionable in the absence of clinical trial data? This question is becoming more frequent, with mainstream media promoting personalized medicine and growing demands from patients to be treated based on the results of next-generation sequencing assays. Whether the decision is to enroll in a clinical trial or to seek off-label use of an already approved drug, these situations are increasingly encountered by practitioners and patients alike.

Identifying actionable driver alterations in routine clinical sequencing assays is not straightforward and can be particularly difficult in uncommon cancer types. Some driver alterations may have well-documented response rates in one histologic type but not in another, thereby making the extrapolation of results of targeted therapies trials from one histology to another not a sound approach. Clinical trials using a basket design have shown mixed results, with not all histologies responding to targeted therapy against a particular mutation. For instance, many BRAF V600E-mutant nonmelanoma histologies respond to vemurafenib; however, BRAF V600E-mutant colorectal cancers did not display any effectiveness with vemurafenib. ${ }^{4}$ This finding suggests that the overall cellular and genomic context in which a driver alteration occurs is important in determining its actionability.

Additionally, a diverse repertoire of mutations can exist in some driver genes that lead to differing biologic consequences for each specific type of mutation. An example of this 
phenomenon is found in the TP53 gene, wherein many well-known truncating mutations cause loss of function of the gene product, but growing evidence demonstrates missense mutations with gain of function properties also occur. ${ }^{5}$ Mutations may also depend on clinical context, especially if the patient has been treated systemically before a tissue specimen was harvested for sequencing. For instance, lung adenocarcinomas with ERRB2 missense mutations at codon 310 are highly associated with the EGFR L858R mutations after anti-EGFR therapy and may represent acquired resistance, but ERRB2 exon 20 insertions are only found in the absence of EGFR alterations and may represent a true founder mutation. ${ }^{6,7}$ We currently do not understand the contexts that predict exceptional responses to targeted inhibition of driver alterations in uncommon cancers. However, we are getting a much better picture of the molecular landscapes of cancer and many insights into the molecular pathology of cancers are accruing.

Genomic databases such as cBioPortal (www.cbiopartal.org) ${ }^{8}$ are providing access to large-scale genomic data where inferences can be made about the actionability of putative driver alterations in a broad set of cancers. However, significant limitations still exist in routine clinical practice to implementing targeted approaches to treat rare driver alterations in uncommon cancer types. One of the largest barriers is intratumoral heterogeneity and the continual somatic genetic evolution that occurs in cancers. Putative driver alterations may look clonal in the sample used for sequencing, but may in fact be subclonal across the whole tumor and render targeted approaches ineffective on the systemic level. The selection of a driver to target can be further confounded by the presence of multiple putative driver alterations occurring in a single cancer specimen. In uncommon cancer types for which we do not have a good molecular landscape of driver alterations, it is unknown which driver is contributing the most selective advantage to the cancer. Hence, predicting which targeted therapy may induce a response is difficult. Furthermore, distinguishing the driver mutation that represents the founder mutation of the cancer from other subclonal driver alterations that have arisen from ongoing mutagenesis and somatic genetic evolution is not entirely possible. Building our knowledge base of driver alterations that occur as the founding mutation and are therefore present in the trunk of a tumor's evolutionary phylogenetic tree is an important stride in the paradigm of personalized medicine.

Recently published results of the TRACERx study ${ }^{9}$ provided great insight into the evolutionary phylogenetic trees of lung cancers. Importantly, this study showed that most of the truncal mutations seen in lung cancers are the well-known founder mutations that are currently tested for as the standard of care. Interestingly, the drivers that occurred rarely in lung adenocarcinoma but are more typical of other histologies were found in subclonal populations that arise during the later evolutionary branches. This finding gives great pause in the decision to target rare driver alterations outside of the histology in which they are typically found. The clonal architecture of lung cancer has also been shown to predict response to immune checkpoint inhibition. Specifically, cancers with many mutations in the trunk of the phylogenetic tree showed a more durable response to PD-1 and CTLA-4 blockade in advanced non-small cell lung cancers and melanoma. ${ }^{10}$ Understanding the clonal architecture of cancers and how cancers undergo genetic evolution will open new opportunities for prognosticating and predicting response to therapy.

Measurements of intratumoral heterogeneity and somatic genetic evolution show great promise for clinical practice. However, we still lack an understanding of the "evolutionary" landscape of the most common cancers and have little ability to assess these factors in routine clinical practice. Future clinical trials using genomic data should incorporate multiregion sequencing of tumors so that we can gain an understanding of how somatic evolution and clonal architecture relate to clinical outcomes and treatment response.

In summary, the case presented by Hamad et al illustrates many of the challenges and questions that arise in applying targeted approaches to rare driver alterations in general and especially uncommon cancer types. Still, it is becoming clear that achieving a meaningful clinical response from targeted therapeutics is increasingly a game of scientific rigor and less of chance. 


\section{References}

1. Gingras MC, Covington KR, Chang DK, et al. Ampullary cancers harbor ELF3 tumor suppressor gene mutations and exhibit frequent WNT dysregulation. Cell Rep 2016;14:907-919.

2. Chan OT, Chen ZM, Chung F, et al. Lack of HER2 overexpression and amplification in small intestinal adenocarcinoma. Am J Clin Pathol 2010;134:880-885.

3. Laforest $\mathrm{A}$, Aparicio $\mathrm{T}$, Zaanan $\mathrm{A}$, et al. ERBB2 gene as a potential therapeutic target in small bowel adenocarcinoma. Eur J Cancer 2014;50:1740-1746.

4. Hyman DM, Puzanov I, Subbiah V, et al. Vemurafenib in multiple nonmelanoma cancers with BRAF V600 mutations. N Engl J Med 2015;373:726-736.

5. Muller PA, Vousden KH. Mutant $p 53$ in cancer: new functions and therapeutic opportunities. Cancer Cell 2014:25:304-317.

6. Shigematsu $H$, Takahashi $T$, Nomura $M$, et al. Somatic mutations of the HER2 kinase domain in lung adenocarcinomas. Cancer Res 2005;65:1642-1646.

7. Gowen K, Halmos B, Hoyer R, et al. MA04.01 Non-amplification mutation of ERBB2 in EGFR-mutated lung cancer. J Thoracic Oncol 2017;12:S357-358.

8. Gao J, Aksoy BA, Dogrusoz U, et al. Integrative analysis of complex cancer genomics and clinical profiles using the cBioPortal. Sci Signal 2013;6:pl1.

9. Jamal-Hanjani M, Wilson GA, McGranahan $\mathrm{N}$, et al. Tracking the evolution of non-small-cell lung cancer. $\mathrm{N}$ Engl J Med 2017;376:2109-2121.

10. McGranahan N, Furness AJ, Rosenthal R, et al. Clonal neoantigens elicit $\mathrm{T}$ cell immunoreactivity and sensitivity to immune checkpoint blockade. Science 2016;351:1463-1469. 